\title{
Bayes approach to study shape parameter of Frechet distribution
}

\author{
Wajiha Nasir*, Muhammad Aslam \\ Department of Basic Sciences Riphah International University Islamabad \\ *Corresponding author E-mail: wajiha.nasir19@gmail.com
}

Copyright (C)2015 Wajiha Nasir, Muhammad Aslam. This is an open access article distributed under the Creative Commons Attribution License, which permits unrestricted use, distribution, and reproduction in any medium, provided the original work is properly cited.

\begin{abstract}
In this paper, Frechet distribution under Bayesian paradigm is studied. Posterior distributions are derived by using Gumbel Type-II and Levy prior. Quadrature numerical integration technique is utilized to solve posterior distribution. Bayes estimators and their risks have been obtained by using four loss functions. Prior predictive distributions are derived for elicitation of hyperparameters. The performance of Bayes estimators are compared by using Monte Carlo simulation study.
\end{abstract}

Keywords: Informative Prior, Bayes Estimates, Posterior Risks, Loss Functions, Elicitation, Monte Carlo simulation.

\section{Introduction}

Frechet distribution has wide application in modeling extreme events such as flood, rainfall, stock exchange and wind speeds etc. [1] shows that the asymptomatic distribution of extreme stocks market return is Frechet distribution. [2] uses Gumbel and Frechet distribution to fit maximum annual wind speeds for the individual stations.

[3] has drived the expressions of estimators of parameter for Frechet Distribution under progressive type-II data using method of maximum likelihood estimation as well as least squares. He also constructs confidence intervals for parameters and percentile for failure time distribution. He has also drived Fisher Information matrix.

[6] has derived estimators of parameters for Frechet distribution using maximum likelihood estimation and least squares under Type-II censored samples. Fisher information matrix and confidence intervals for parameters are also constructed and simulation technique for the purpose of comparison is utilized.[5] has found out the estimator of scale parameter using Maximum Likelihood Estimation, probability weighted Moment and Bayesian estimation methods by assuming known shape parameter of Frechet distribution. Jeffreys prior and three loss functions has used to find out posterior distribution and Bayes estimators of parameter respectively.

[8] has studied Frechet distribution with known shape parameter by using different sampling schemes. They has derived maximum likelihood estimator of scale parameter and posterior distributions under non-informative priors (Uniform and Jeffreys prior) by using complete, and censoring techniques (type-I and type-II) has derived using square error and precautionary loss functions.

The p.d.f for Frechet distribution is

$f(\mathrm{x} ; \alpha, \beta)=\alpha \beta^{\alpha}\left(\frac{1}{\mathrm{x}}\right)^{\alpha+1} e^{-\left(\frac{\beta}{\mathrm{x}}\right)^{\alpha}} \quad 0<x<\infty, 0<\alpha<\infty, 0<\beta<\infty$, 
where $\alpha$ is shape parameter and $\beta$ is scale parameter.

We will study Bayes estimators of shape parameter with known shape parameter of Frechet distribution . We will use four loss functions using Gumbel type-II and Levy prior.

\section{Loss Functions}

This section contains Bayes estimators and posterior risks of different loss functions.

Some LF are defined below:

1. Square Error Loss Function (SELF)

The square error loss function is given as

$L\left(\alpha, \alpha^{*}\right)=\left(\alpha-\alpha^{*}\right)^{2}$

where $\alpha^{*}$ is the estimator of parameter $\alpha$.

Now the Bayes estimator is obtained by minimizing the expectation w.r.t. posterior distribution of the loss function

$\alpha^{*}=E(\alpha)$,

and the Bayes posterior risk is obtained as

$\rho\left(\alpha^{*}\right)=E_{\alpha \mid \mathbf{x}} \quad\left\{L\left(\alpha, \alpha^{*}\right)\right\}=\operatorname{Var}(\alpha)$.

2. Simple Precautionary Loss Function (SPLF)

[10] introduced the following Precautionary Loss Function with quadratic loss function as a special case.

$L\left(\alpha, \alpha^{*}\right)=\left(\frac{\alpha-\alpha^{*}}{\alpha^{*}}\right)^{2}$,

where $\alpha^{*}$ is the estimator of parameter $\alpha$.

Now the Bayes estimator is obtained by minimizing the expectation w.r.t. posterior distribution of the loss function

$\alpha^{*}=\sqrt{\frac{E(\alpha \mid \mathrm{x})}{E\left(\alpha^{-1} \mid \mathrm{x}\right)}}$.

And the Bayes posterior risk is obtained as

$\rho\left(\alpha^{*}\right)=2 *\left[\sqrt{E\left(\alpha^{-1} \mid \mathrm{x}\right) * E(\alpha \mid \mathrm{x})}-1\right]$.

\section{Weighted Balanced Loss Function}

The weighted balanced loss function is defined as

$L\left(\alpha, \alpha^{*}\right)=\left(\frac{\alpha-\alpha^{*}}{\alpha^{*}}\right)^{2}$,

where $\alpha^{*}$ is the estimator of parameter $\alpha$ Now the Bayes estimator is obtained by minimizing the expectation w.r.t. posterior distribution of the loss function

$\alpha^{*}=\frac{E\left(\alpha^{2} \mid \mathrm{x}\right)}{E(\alpha \mid \mathrm{x})}$,

and the Bayes posterior risk is obtained as

$\rho\left(\alpha^{*}\right)=1-\frac{[E(\alpha \mid \mathrm{x})]^{2}}{E\left(\alpha^{2} \mid \mathrm{x}\right)}$. 


\section{Minimum Expected Loss Function (MELF)}

In Bayesian analysis, widely used loss function is a quadratic loss function given below

$L\left(\alpha, \alpha^{*}\right)=w\left(\alpha^{*}-\alpha\right)^{2}$,

where $\alpha^{*}$ is the estimator of parameter $\alpha$.

If $w=1$, then it reduces to square error loss function and for $w=\alpha^{-2}$ it becomes

$L\left(\alpha, \alpha^{*}\right)=\frac{\left(\alpha^{*}-\alpha\right)^{2}}{\alpha^{2}}$,

known as MELF introduced by [9] in their study.

Now the Bayes estimator is obtained by minimizing the expectation w.r.t. posterior distribution of the loss function

$\alpha^{*}=\frac{E\left(\alpha^{-1} \mid \mathrm{x}\right)}{E\left(\alpha^{-2} \mid \mathrm{x}\right)}$

, and the Bayes posterior risk is obtained as

$\rho\left(\alpha^{*}\right)=1-\frac{\left[E\left(\alpha^{-1} \mid \mathrm{x}\right)\right]^{2}}{E\left(\alpha^{-2} \mid \mathrm{x}\right)}$.

\section{Posterior Distribution}

Posterior distribution is derived by using Gumbel Type-II and Levy prior.

\subsection{Posterior Distribution using Gumbel Type-II prior}

The Gumbel Type-II with hyperparameters $c$ is

$p(\alpha)=\frac{c}{\alpha^{2}} e^{-\frac{c}{\alpha}}, \quad 0<\alpha<\infty, \quad c>0$.

The posterior distribution of shape parameter for given data using $\left(x=x_{1}, x_{2}, \ldots, x_{n}\right)$ is:

$p(\alpha \mid \mathbf{x})=\frac{\alpha^{n-2} \beta^{n \alpha} \prod_{i=1}^{n}\left(\frac{1}{x_{i}}\right)^{\alpha+1} e^{-\frac{c}{\alpha}-\beta^{\alpha} \sum_{i=1}^{n}\left(\frac{1}{x_{i}}\right)^{\alpha}}}{k_{1}}$,

where

$k_{1}=\int_{0}^{\infty} \alpha^{n-2} \beta^{n \alpha} \prod_{i=1}^{n}\left(\frac{1}{x_{i}}\right)^{\alpha+1} e^{-\frac{c}{\alpha}-\beta^{\alpha} \sum_{i=1}^{n}\left(\frac{1}{x_{i}}\right)^{\alpha}} d \alpha$.

which is solved by numerical integration technique.

The Bayes estimators using SELF is

$\hat{\alpha}=\int_{0}^{\infty} \frac{\alpha^{n-1}}{k_{1}} \beta^{n \alpha} \prod_{i=1}^{n}\left(\frac{1}{x_{i}}\right)^{\alpha} e^{-\left(\frac{\beta}{x_{i}}\right)^{\alpha}} d \alpha$.

The posterior risks using SELF is

$\rho(\hat{\alpha})=\int_{0}^{\infty} \frac{\alpha^{n}}{k_{1}} \beta^{n \alpha} \prod_{i=1}^{n}\left(\frac{1}{x_{i}}\right)^{\alpha} e^{-\frac{c}{\alpha}-\left(\frac{\beta}{x_{i}}\right)^{\alpha}} d \alpha-\left(\int_{0}^{\infty} \frac{\alpha^{n-1}}{k_{1}} \beta^{n \alpha} \prod_{i=1}^{n}\left(\frac{1}{x_{i}}\right)^{\alpha} e^{-\frac{c}{\alpha}-\left(\frac{\beta}{x_{i}}\right)^{\alpha}} d \alpha\right)^{2}$. 
The Bayes estimators using SPLF is

$\hat{\alpha}=\sqrt{\frac{\int_{0}^{\infty} \alpha^{n-1} \beta^{n \alpha} \prod_{i=1}^{n}\left(\frac{1}{x_{i}}\right)^{\alpha} e^{-\frac{c}{\alpha}-\left(\frac{\beta}{x_{i}}\right)^{\alpha}} d \alpha}{\int_{0}^{\infty} \alpha^{n-3} \beta^{n \alpha} \prod_{i=1}^{n}\left(\frac{1}{x_{i}}\right)^{\alpha} e^{-\frac{c}{\alpha}-\left(\frac{\beta}{x_{i}}\right)^{\alpha}} d \alpha} .}$

The posterior risks using SPLF is

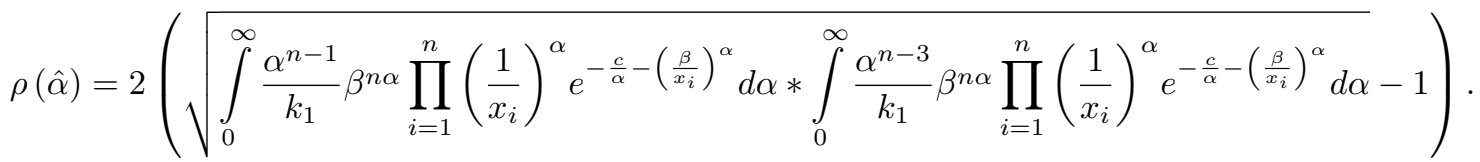

The Bayes estimators using WBLF is

$\hat{\alpha}=\frac{\int_{0}^{\infty} \alpha^{n-1} \beta^{n \alpha} \prod_{i=1}^{n}\left(\frac{1}{x_{i}}\right)^{\alpha} e^{-\frac{c}{\alpha}-\left(\frac{\beta}{x_{i}}\right)^{\alpha}} d \alpha}{\int_{0}^{\infty} \alpha^{n} \beta^{n \alpha} \prod_{i=1}^{n}\left(\frac{1}{x_{i}}\right)^{\alpha} e^{-\frac{c}{\alpha}-\left(\frac{\beta}{x_{i}}\right)^{\alpha}} d \alpha}$.

The posterior risks using WBLF is

$\rho(\hat{\alpha})=1-\frac{\left(\int_{0}^{\infty} \alpha^{n-1} \beta^{n \alpha} \prod_{i=1}^{n}\left(\frac{1}{x_{i}}\right)^{\alpha} e^{-\frac{c}{\alpha}-\left(\frac{\beta}{x_{i}}\right)^{\alpha}} d \alpha\right)^{2}}{\int_{0}^{\infty} \alpha^{n} \beta^{n \alpha} \prod_{i=1}^{n}\left(\frac{1}{x_{i}}\right)^{\alpha} e^{-\frac{c}{\alpha}-\left(\frac{\beta}{x_{i}}\right)^{\alpha}} d \alpha}$.

The Bayes estimators using MELF is

$\hat{\alpha}=\frac{\int_{0}^{\infty} \alpha^{n-3} \beta^{n \alpha} \prod_{i=1}^{n}\left(\frac{1}{x_{i}}\right)^{\alpha} e^{-\frac{c}{\alpha}-\left(\frac{\beta}{x_{i}}\right)^{\alpha}} d \alpha}{\int_{0}^{\infty} \alpha^{n-4} \beta^{n \alpha} \prod_{i=1}^{n}\left(\frac{1}{x_{i}}\right)^{\alpha} e^{-\frac{c}{\alpha}-\left(\frac{\beta}{x_{i}}\right)^{\alpha}} d \alpha}$.

The posterior risks using MELF is

$\rho(\hat{\alpha})=1-\frac{\left(\int_{0}^{\infty} \alpha^{n-3} \beta^{n \alpha} \prod_{i=1}^{n}\left(\frac{1}{x_{i}}\right)^{\alpha} e^{-\frac{c}{\alpha}-\left(\frac{\beta}{x_{i}}\right)^{\alpha}} d \alpha\right)^{2}}{\int_{0}^{\infty} \alpha^{n-4} \beta^{n \alpha} \prod_{i=1}^{n}\left(\frac{1}{x_{i}}\right)^{\alpha} e^{-\frac{c}{\alpha}-\left(\frac{\beta}{x_{i}}\right)^{\alpha}} d \alpha}$.

\subsection{Posterior distribution using Levy Prior}

The Levy prior with hyperparameter $l$ is

$p(\alpha)=\sqrt{\frac{l}{2 \pi}} \alpha^{-\frac{3}{2}} e^{\frac{-l}{2 \alpha}}, \quad 0<\alpha<\infty, \quad t>0$.

The posterior distribution of shape parameter is:

$(\alpha \mid \mathbf{x})=\frac{\alpha^{n-\frac{3}{2}} \beta^{\alpha n} e^{-\frac{l}{2 \alpha}-\beta^{\alpha} \sum_{i=1}^{n}\left(\frac{1}{x_{i}}\right)^{\alpha}}}{k_{4}}$,

where

$k_{4}=\int_{0}^{\infty} \alpha^{n-\frac{3}{2}} \beta^{\alpha n} \prod_{i=1}^{n}\left(\frac{1}{x_{i}}\right)^{\alpha+1} e^{-\frac{l}{2 \beta}-\beta^{\alpha} \sum_{i=1}^{n}\left(\frac{1}{x_{i}}\right)^{\alpha}} d \alpha$. 
which is solved by numerical integration technique.

The Bayes estimators using SELF is

$\hat{\alpha}=\int_{0}^{\infty} \frac{\alpha^{n-\frac{5}{2}}}{k_{2}} \beta^{n \alpha} \prod_{i=1}^{n}\left(\frac{1}{x_{i}}\right)^{\alpha} e^{-\frac{l}{2 \beta}-\left(\frac{\beta}{x_{i}}\right)^{\alpha}} d \alpha$.

The posterior risks using SELF is

$\rho(\hat{\alpha})=\int_{0}^{\infty} \frac{\alpha^{n-\frac{1}{2}}}{k_{2}} \beta^{n \alpha} \prod_{i=1}^{n}\left(\frac{1}{x_{i}}\right)^{\alpha} e^{-\frac{l}{2 \beta}-\left(\frac{\beta}{x_{i}}\right)^{\alpha}} d \alpha-\left(\int_{0}^{\infty} \frac{\alpha^{n+\frac{1}{2}}}{k_{2}} \beta^{n \alpha} \prod_{i=1}^{n}\left(\frac{1}{x_{i}}\right)^{\alpha} e^{-\frac{l}{2 \beta}-\left(\frac{\beta}{x_{i}}\right)^{\alpha}} d \alpha\right)^{2}$.

The Bayes estimators using SPLF is

$\hat{\alpha}=\sqrt{\frac{\int_{0}^{\infty} \alpha^{n-\frac{1}{2}} \prod_{i=1}^{n}\left(\frac{1}{x_{i}}\right)^{\alpha} e^{-\frac{l}{2 \beta}-\left(\frac{\beta}{x_{i}}\right)^{\alpha}} d \alpha}{\int_{0}^{\infty} \alpha^{n-\frac{5}{2}} \prod_{i=1}^{n}\left(\frac{1}{x_{i}}\right)^{\alpha} e^{-\frac{l}{2 \beta}-\left(\frac{\beta}{x_{i}}\right)^{\alpha}} d \alpha} .}$

The posterior risks using SPLF is

$\hat{\alpha}=\sqrt{\frac{\int_{0}^{\infty} \alpha^{n-\frac{1}{2}} \prod_{i=1}^{n}\left(\frac{1}{x_{i}}\right)^{\alpha} e^{-\frac{l}{2 \beta}-\left(\frac{\beta}{x_{i}}\right)^{\alpha}} d \alpha}{\int_{0}^{\infty} \alpha^{n-\frac{5}{2}} \prod_{i=1}^{n}\left(\frac{1}{x_{i}}\right)^{\alpha} e^{-\frac{l}{2 \beta}-\left(\frac{\beta}{x_{i}}\right)^{\alpha}} d \alpha} .}$

The Bayes estimators using SPLF is

$\hat{\alpha}=\frac{\int_{0}^{\infty} \alpha^{n-\frac{1}{2}} \beta^{n \alpha} \prod_{i=1}^{n}\left(\frac{1}{x_{i}}\right)^{\alpha} e^{-\frac{l}{2 \beta}-\left(\frac{\beta}{x_{i}}\right)^{\alpha}} d \alpha}{\int_{0}^{\infty} \alpha^{n+\frac{1}{2}} \beta^{n \alpha} \prod_{i=1}^{n}\left(\frac{1}{x_{i}}\right)^{\alpha} e^{-\frac{l}{2 \beta}-\left(\frac{\beta}{x_{i}}\right)^{\alpha}} d \alpha}$.

The posterior risks using WBLF is

$\rho(\hat{\alpha})=1-\frac{\left(\int_{0}^{\infty} \alpha^{n-\frac{1}{2}} \beta^{n \alpha} \prod_{i=1}^{n}\left(\frac{1}{x_{i}}\right)^{\alpha} e^{-\frac{l}{2 \beta}-\left(\frac{\beta}{x_{i}}\right)^{\alpha}} d \alpha\right)^{2}}{\int_{0}^{\infty} \alpha^{n+\frac{1}{2}} \beta^{n \alpha} \prod_{i=1}^{n}\left(\frac{1}{x_{i}}\right)^{\alpha} e^{-\frac{l}{2 \beta}-\left(\frac{\beta}{x_{i}}\right)^{\alpha}} d \alpha}$.

The Bayes estimators using MELF is

$\hat{\alpha}=\frac{\int_{0}^{\infty} \alpha^{n-\frac{5}{2}} \beta^{n \alpha} \prod_{i=1}^{n}\left(\frac{1}{x_{i}}\right)^{\alpha} e^{-\frac{l}{2 \beta}-\left(\frac{\beta}{x_{i}}\right)^{\alpha}} d \alpha}{\int_{0}^{\infty} \alpha^{n-\frac{7}{2}} \beta^{n \alpha} \prod_{i=1}^{n}\left(\frac{1}{x_{i}}\right)^{\alpha} e^{-\frac{l}{2 \beta}-\left(\frac{\beta}{x_{i}}\right)^{\alpha}} d \alpha}$.

The posterior risks using MELF is

$\rho(\hat{\alpha})=1-\frac{\left(\int_{0}^{\infty} \alpha^{n-\frac{5}{2}} \beta^{n \alpha} \prod_{i=1}^{n}\left(\frac{1}{x_{i}}\right)^{\alpha} e^{-\frac{l}{2 \beta}-\left(\frac{\beta}{x_{i}}\right)^{\alpha}} d \alpha\right)^{2}}{\int_{0}^{\infty} \alpha^{n-\frac{7}{2}} \beta^{n \alpha} \prod_{i=1}^{n}\left(\frac{1}{x_{i}}\right)^{\alpha} e^{-\frac{l}{2 \beta}-\left(\frac{\beta}{x_{i}}\right)^{\alpha}} d \alpha}$.

\section{Elicitation of Hyper-parameters}

We used the method for elicitation of hyperparameters by using prior predictive distributions proposed by [7]. Prior predictive distribution is derived by

$p(y)=\int_{-\infty}^{\infty} p(\alpha) f(x ; \alpha, \beta) d \alpha$ 


\subsection{Prior predictive using Gumbel Type-II Prior}

The prior predictive distribution of future random variable $\mathrm{Y}$ using eq 37 is

$p(y)=\int_{0}^{\infty} \frac{c}{\alpha} \beta^{a}\left(\frac{1}{y}\right)^{\alpha} e^{-\left(\frac{\beta}{y}\right)^{\alpha}-\frac{c}{\alpha}} d \alpha$ where $y>0$.

By the method of elicitation the value for $c$ is 0.996577 .

\subsection{Prior predictive using Levy Prior}

The prior predictive distribution of future random variable $\mathrm{Y}$ using eq 37 is

$p(y)=\int_{0}^{\infty} \sqrt{\frac{l}{2 \alpha \pi}} \beta^{a}\left(\frac{1}{y}\right)^{\alpha} e^{-\left(\frac{\beta}{y}\right)^{\alpha}-\frac{l}{2 \alpha}} d \alpha$ where $y>0$.

By the method of elicitation the value for $l$ is 1.098929 .

\section{Simulation Study}

We use simulation to check the performance of prior distributions and loss functions. For this purpose We use different values of shape parameter by using different sample sizes. We use Mathimatica 10 package to generate random sample of size $n=\{30,50,70,100\}$ form Frechet distribution by using values of $\alpha \in\{5,8\}$ and $\beta \in\{2,3\}$. The Bayes posterior risks are in parentheses. The results for simulation study are summarized in the following tables

Table 1: Simulation of Bayes estimators and posterior risks under SELF using different Priors

\begin{tabular}{|c|l|l|l|l|l|l|l|l|}
\hline $\mathrm{n}$ & 30 & 50 & 70 & 100 & 30 & 50 & 70 & 100 \\
\hline & \multicolumn{5}{|c|}{$\alpha=2, \beta=5$} & \multicolumn{5}{|c|}{$\alpha=3, \beta=5$} \\
\hline UP & 2.0897 & 2.0516 & 2.0378 & 2.0336 & 3.1009 & 3.0621 & 3.0529 & 3.0198 \\
& $(0.0809)$ & $(0.0466)$ & $(0.0328)$ & $(0.0228)$ & $(0.1773)$ & $(0.1039)$ & $(0.0737)$ & $(0.0503)$ \\
\hline JP & 2.1113 & 2.0658 & 2.0419 & 2.0375 & 3.1889 & 3.1159 & 3.0775 & 3.0617 \\
& $(0.0801)$ & $(0.0462)$ & $(0.0324)$ & $(0.0226)$ & $(0.1722)$ & $(0.1035)$ & $(0.0736)$ & $(0.0500)$ \\
\hline GTP & 2.0270 & 2.0312 & 2.0157 & 2.0130 & 3.0890 & 3.0624 & 3.0452 & 3.06017 \\
& $(0.0671)$ & $(0.0392)$ & $(0.0270)$ & $(0.0187)$ & $(0.1601)$ & $(0.0909)$ & $(0.0633)$ & $(0.0443)$ \\
\hline LP & 2.0928 & 2.0855 & 2.0694 & 2.0644 & 3.0047 & 3.0843 & 3.0755 & 3.0869 \\
& $(0.0711)$ & $(0.0414)$ & $(0.0285)$ & $(0.0198)$ & $(0.1657)$ & $(0.0953)$ & $(0.0667)$ & $(0.0467)$ \\
\hline \multirow{6}{|c|}{$\alpha=2, \beta=8$} \\
UP & 2.0908 & 2.0483 & 2.0330 & 2.0239 & 3.11271 & 3.0763 & 3.0456 & 3.0267 \\
& $(0.0810)$ & $(0.0465)$ & $(0.0325)$ & $(0.0225)$ & $(0.1781)$ & $(0.1044)$ & $(0.0733)$ & $(0.0506)$ \\
\hline JP & 2.1072 & 2.0691 & 2.0444 & 2.0379 & 3.1895 & 3.1109 & 3.0787 & 3.0556 \\
& $(0.0795)$ & $(0.0464)$ & $(0.0323)$ & $(0.0225)$ & $(0.1735)$ & $(0.1040)$ & $(0.0732)$ & $(0.0502)$ \\
\hline GTP & 2.0931 & 2.0888 & 2.0842 & 2.0822 & 3.0480 & 3.5324 & 3.0231 & 3.0035 \\
& $(0.0672)$ & $(0.0382)$ & $(0.0267)$ & $(0.0186)$ & $(0.1569)$ & $(0.0898)$ & $(0.0626)$ & $(0.0427)$ \\
\hline LP & 2.0998 & 2.0732 & 2.0691 & 2.0636 & 3.0965 & 3.0923 & 3.02861 & 3.0686 \\
& $(0.0721)$ & $(0.0409)$ & $(0.0287)$ & $(0.0198)$ & $(0.1636)$ & $(0.0964)$ & $(0.0671)$ & $(0.0461)$ \\
\hline
\end{tabular}


Table 2: Simulation of Bayes estimators and posterior risks under SPLF using different Priors

\begin{tabular}{|c|l|l|l|l|l|l|l|l|}
\hline $\mathrm{n}$ & 30 & 50 & 70 & 100 & 30 & 50 & 70 & 100 \\
\hline & \multicolumn{5}{|c|}{$\alpha=2, \beta=5$} & \multicolumn{4}{|c|}{$\alpha=3, \beta=5$} \\
\hline UP & 2.0589 & 2.0483 & 2.0188 & 2.0165 & 3.0863 & 3.0461 & 3.0515 & 3.0160 \\
& $(0.0187)$ & $(0.0112)$ & $(0.0079)$ & $(0.0055)$ & $(0.0186)$ & $(0.0111)$ & $(0.0079)$ & $(0.0055)$ \\
\hline JP & 2.1124 & 2.0554 & 2.0393 & 2.0256 & 3.1561 & 3.0983 & 3.0656 & 3.0472 \\
& $(0.0180)$ & $(0.0109)$ & $(0.0078)$ & $(0.0054)$ & $(0.0179)$ & $(0.0108)$ & $(0.0077)$ & $(0.0054)$ \\
\hline GTP & 2.0077 & 2.0161 & 2.0130 & 2.0082 & 3.0361 & 3.0508 & 3.0304 & 3.0267 \\
& $(0.0112)$ & $(0.0065)$ & $(0.0046)$ & $(0.0032)$ & $(0.0130)$ & $(0.0075)$ & $(0.0053)$ & $(0.0036)$ \\
\hline LP & 2.0960 & 2.0846 & 2.0605 & 2.0706 & 3.0848 & 3.0708 & 3.0639 & 3.0658 \\
& $(0.0134)$ & $(0.0078)$ & $(0.0055)$ & $(0.0038)$ & $(0.0151)$ & $(0.0088)$ & $(0.0062)$ & $(0.0043)$ \\
\hline \multirow{6}{|c|}{$\alpha=2, \beta=8$} \\
UP & 2.0631 & 2.0384 & 2.0244 & 2.0124 & 3.1073 & 3.0516 & 3.0157 & 3.0345 \\
& $(0.0188)$ & $(0.0111)$ & $(0.0079)$ & $(0.0055)$ & $(0.0188)$ & $(0.0111)$ & $(0.0079)$ & $(0.0055)$ \\
\hline JP & 2.0990 & 2.0430 & 2.0358 & 2.0342 & 3.1405 & 3.0783 & 3.0574 & 3.0309 \\
& $(0.0181)$ & $(0.0109)$ & $(0.0077)$ & $(0.0054)$ & $(0.0179)$ & $(0.0109)$ & $(0.0078)$ & $(0.0054)$ \\
\hline GTP & 2.0923 & 2.0802 & 2.0849 & 2.0646 & 3.0305 & 3.0307 & 3.0260 & 3.0195 \\
& $(0.0104)$ & $(0.0060)$ & $(0.0043)$ & $(0.0029)$ & $(0.0122)$ & $(0.0071)$ & $(0.0050)$ & $(0.0034)$ \\
\hline LP & 2.0799 & 2.0762 & 2.0635 & 2.0657 & 3.0692 & 3.0779 & 3.0791 & 3.0665 \\
& $(0.0135)$ & $(0.0078)$ & $(0.0055)$ & $(0.0038)$ & $(0.0150)$ & $(0.0088)$ & $(0.0062)$ & $(0.0043)$ \\
\hline
\end{tabular}

Table 3: Simulation of Bayes estimators and posterior risks under WBLF using different Priors

\begin{tabular}{|c|l|l|l|l|l|l|l|l|}
\hline $\mathrm{n}$ & 30 & 50 & 70 & 100 & 30 & 50 & 70 & 100 \\
\hline & \multicolumn{5}{|c|}{$\alpha=2, \beta=5$} & \multicolumn{4}{|c|}{$\alpha=3, \beta=5$} \\
\hline UP & 2.1149 & 2.0786 & 2.0506 & 2.1149 & 2.0786 & 2.0506 & 2.1149 & 2.0786 \\
& $(0.0176)$ & $(0.0108)$ & $(0.0077)$ & $(0.0176)$ & $(0.0108)$ & $(0.0077)$ & $(0.0176)$ & $(0.0108)$ \\
\hline JP & 2.1510 & 2.0860 & 2.0642 & 2.1510 & 2.0860 & 2.0642 & 2.1510 & 2.0860 \\
& $(0.0173)$ & $(0.0105)$ & $(0.0076)$ & $(0.0173)$ & $(0.0105)$ & $(0.0076)$ & $(0.0173)$ & $(0.0105)$ \\
\hline GTP & 2.0660 & 2.0501 & 2.0308 & 2.0660 & 2.0501 & 2.0308 & 2.0660 & 2.0501 \\
& $(0.0108)$ & $(0.0064)$ & $(0.0045)$ & $(0.0108)$ & $(0.0064)$ & $(0.0045)$ & $(0.0108)$ & $(0.0064)$ \\
\hline LP & 2.0327 & 2.0184 & 2.0964 & 2.0327 & 2.0184 & 2.0964 & 2.0327 & 2.0184 \\
& $(0.0130)$ & $(0.0077)$ & $(0.0054)$ & $(0.0130)$ & $(0.0077)$ & $(0.0054)$ & $(0.0130)$ & $(0.0077)$ \\
\hline \multirow{6}{|c|}{$\alpha=2, \beta=8$} \\
UP & 2.1201 & 2.0652 & 2.0524 & 2.1201 & 2.0652 & 2.0524 & 2.1201 & 2.0652 \\
& $(0.0177)$ & $(0.0108)$ & $(0.0077)$ & $(0.0177)$ & $(0.0108)$ & $(0.0077)$ & $(0.0177)$ & $(0.0108)$ \\
\hline JP & 2.1491 & 2.0936 & 2.0689 & 2.1491 & 2.0936 & 2.0689 & 2.1491 & 2.0936 \\
& $(0.0172)$ & $(0.0106)$ & $(0.0076)$ & $(0.0172)$ & $(0.0106)$ & $(0.0076)$ & $(0.0172)$ & $(0.0106)$ \\
\hline GTP & 2.0337 & 2.0869 & 2.0838 & 2.0337 & 2.0869 & 2.0838 & 2.0337 & 2.0869 \\
& $(0.0101)$ & $(0.0059)$ & $(0.0042)$ & $(0.0101)$ & $(0.0059)$ & $(0.0042)$ & $(0.0101)$ & $(0.0059)$ \\
\hline LP & 2.0261 & 2.0963 & 2.0824 & 2.0261 & 2.0963 & 2.0824 & 2.0261 & 2.0963 \\
& $(0.0130)$ & $(0.0077)$ & $(0.0054)$ & $(0.0130)$ & $(0.0077)$ & $(0.0054)$ & $(0.0130)$ & $(0.0077)$ \\
\hline
\end{tabular}


Table 4: Simulation of Bayes estimators and posterior risks under MELF using different Priors

\begin{tabular}{|c|l|l|l|l|l|l|l|l|}
\hline $\mathrm{n}$ & 30 & 50 & 70 & 100 & 30 & 50 & 70 & 100 \\
\hline & \multicolumn{5}{|c|}{$\alpha=2, \beta=5$} & \multicolumn{5}{|c|}{$\alpha=3, \beta=5$} \\
\hline UP & 2.0068 & 1.9976 & 2.0050 & 2.0034 & 2.9866 & 2.9892 & 2.9945 & 3.0052 \\
& $(0.0191)$ & $(0.0112)$ & $(0.0080)$ & $(0.0055)$ & $(0.0191)$ & $(0.0113)$ & $(0.0080)$ & $(0.0055)$ \\
\hline JP & 2.0468 & 2.0238 & 2.0170 & 2.0035 & 3.0535 & 3.0287 & 3.0049 & 3.0214 \\
& $(0.0185)$ & $(0.0110)$ & $(0.0078)$ & $(0.0055)$ & $(0.0186)$ & $(0.0110)$ & $(0.0078)$ & $(0.0054)$ \\
\hline GTP & 2.0842 & 2.0983 & 2.0918 & 2.0951 & 3.0860 & 3.0994 & 3.0198 & 3.0178 \\
& $(0.0114)$ & $(0.0065)$ & $(0.0046)$ & $(0.0032)$ & $(0.0131)$ & $(0.0076)$ & $(0.0053)$ & $(0.0037)$ \\
\hline LP & 2.0281 & 2.0474 & 2.0472 & 2.0487 & 3.0411 & 3.0419 & 3.0546 & 3.0481 \\
& $(0.0135)$ & $(0.0079)$ & $(0.0056)$ & $(0.0038)$ & $(0.0153)$ & $(0.0088)$ & $(0.0062)$ & $(0.0043)$ \\
\hline \multirow{6}{*}{ UP } & 1.9876 & 1.9971 & 2.0085 & 2.0046 & 2.9981 & 2.9984 & 3.0189 & 3.0159 \\
& $(0.0192)$ & $(0.0112)$ & $(0.0080)$ & $(0.0055)$ & $(0.0193)$ & $(0.0113)$ & $(0.0080)$ & $(0.0055)$ \\
\hline JP & 2.0457 & 2.0315 & 2.0145 & 2.0091 & 3.0438 & 3.0172 & 3.0169 & 3.0215 \\
& $(0.0184)$ & $(0.0110)$ & $(0.0078)$ & $(0.0055)$ & $(0.0185)$ & $(0.0109)$ & $(0.0078)$ & $(0.0055)$ \\
\hline GTP & 2.0433 & 2.0589 & 2.0589 & 2.0584 & 3.0670 & 3.0816 & 3.0952 & 3.0924 \\
& $(0.0105)$ & $(0.0061)$ & $(0.0043)$ & $(0.0029)$ & $(0.0124)$ & $(0.0071)$ & $(0.0050)$ & $(0.0034)$ \\
\hline LP & 2.0270 & 2.0520 & 2.0494 & 2.0536 & 3.0909 & 3.0383 & 3.0445 & 3.0498 \\
& $(0.0136)$ & $(0.0079)$ & $(0.0055)$ & $(0.0038)$ & $(0.0152)$ & $(0.0089)$ & $(0.0062)$ & $(0.0043)$ \\
\hline
\end{tabular}

\section{Conclusion}

From simulation study, it is concluded that Bayes estimates approaches to their true parameter and posterior risks is decreases by increasing sample size. Gumbel type-II prior is performing better than Levy prior as its posterior risks is smallest among two assumed prior distributions. Weighted balanced loss function is performing better as its posterior risks is least among all loss functions. So, Gumbel type-II with weighted balanced loss function provides less posterior risks as compare to other loss functions and prior.

The study can be further extended by using others loss functions and prior distributions.

\section{References}

[1] Francois M Longin, "The asymptotic distribution of extreme stock market returns.", Journal of business,(1996), pp.383408.

[2] Diwinder Kaur Arora, Er Avadhesh Kumar Maurya, and Ram Asrey Gautam., " Exact modelling and analytical study of annual maximum rainfall with Gumbel and Frechet distributions using parameter estimation techniques..", World of Sciences Journal,Vol.2,(2013), pp.11-26.

[3] Mohamed Mubarak., "Parameter estimation based on the frechet progressive type ii censored data with binomial removals", International Journal of Quality, Statistics, and Reliability, (2012)

[4] Alexander McFarlane Mood, Introduction to the theory of statistics, (1950)

[5] Kamran Abbas and Yincai Tang., "Estimation of parameters for frechet distribution based on type-ii censored samples", Caspian Journal of Applied Sciences Research, $\operatorname{Vol}(2)$, (2013)

[6] Kamran Abbas and Yincai Tang., "Comparison of estimation methods for frechet distribution with known shape", Caspian Journal of Applied Sciences Research,Vol(1), (2012)

[7] Muhammad Aslam.,An application of prior predictive distribution to elicit the prior density, Journal of Statistical Theory and Applications, $\operatorname{Vol}(1),(2003)$ 70-83.

[8] Sindhu Tabassum Naz, Muhammad Aslam, Navid Feroze, On estimation of the frechet distribution with known shape under different sample schemes., International Journal of Intelligent Technologies and Applied Statistics, Vol(7), (2004), $353-358$

[9] VM Tummala and Prakash T Sathe., Minimum expected loss estimators of reliability and parameters of certain lifetime distributions, Reliability, IEEE Transactions on, (1978), Vol(27),283-285 
[10] Jan Gerhard Norstrom, The use of precautionary loss functions in risk analysis, Reliability, IEEE Transactions on, (1996), $\operatorname{Vol}(3), 400-403$ 